\title{
Periodic solution of a quasilinear parabolic equation with nonlocal terms and Neumann boundary conditions
}

\author{
Raad Awad Hameed ${ }^{1,2}$, Boying Wu' and Jiebao Sun ${ }^{1 *}$
}

"Correspondence:

sunjiebao@hit.edu.cn

'Department of Mathematics,

Harbin Institute of Technology,

Harbin, 150001, P.R. China

Full list of author information is

available at the end of the article

\begin{abstract}
In this article, we study the periodic solution of a quasilinear parabolic equation with nonlocal terms and Neumann boundary conditions. By using the theory of Leray-Schauder degree, we obtain the existence of a nontrivial nonnegative time periodic solution.
\end{abstract}

\section{Introduction}

The aim of this work is to consider the following periodic problem for a quasilinear parabolic equation:

$$
\begin{aligned}
& \frac{\partial u}{\partial t}-D_{i}\left(a_{i j}(x, t, u) D_{j} u\right)=(m-\Phi[u]) u, \quad(x, t) \in Q_{T}, \\
& \frac{\partial u}{\partial n}=0, \quad(x, t) \in \partial \Omega \times(0, T), \\
& u(x, 0)=u(x, T), \quad x \in \Omega,
\end{aligned}
$$

where $\Omega$ is a bounded domain in $\mathbb{R}^{n}$ with smooth boundary $\partial \Omega, \frac{\partial}{\partial n}$ denotes the outward normal derivative on $\partial \Omega, Q_{T}=\Omega \times(0, T), a_{i j}$ satisfies some suitable smoothness and structure conditions. This model can be used to describe the models for some interesting phenomena in mathematical biology, fisheries and wildlife management. The function $u(x, t)$ gives the number of individuals (per unit area) of the species at position $x$ and time $t$, where $x$ represents the spatial variable and $t$ represents the time. The term $D_{i}\left(a_{i j}(x, t, u) D_{j} u\right)$ models a tendency to avoid high density in the habitat, $m-\Phi[u]$ describes the ways in which a given population grows and shrinks over time, as controlled by birth, death, emigration or immigration, and the Neumann boundary condition models the trend of the biology population who survive on the boundary.

In last decades, linear parabolic equations with nonlocal terms have been investigated by numerous researchers [1-4]. A typical model was submitted by Allegretto and Nistri [1] and they proposed the following equation:

$$
\frac{\partial u}{\partial t}-\Delta u=f(x, t, m, \Phi[u], u)
$$

with the Dirichlet boundary conditions. Also, according to the actual needs, many authors

\section{Springer}

(c) 2013 Hameed et al.; licensee Springer. This is an Open Access article distributed under the terms of the Creative Commons Attribution License (http://creativecommons.org/licenses/by/2.0), which permits unrestricted use, distribution, and reproduction in any medium, provided the original work is properly cited. 
divert attention to nonlinear diffusion equations with nonlocal terms such as the porous equation $[5,6]$ with a typical form

$$
\frac{\partial u}{\partial t}=\Delta u^{m}+(m-\Phi[u]) u,
$$

and the $p$-Laplacian equation [7] with a typical form

$$
\frac{\partial u}{\partial t}=\operatorname{div}\left(|\nabla u|^{p-2} \nabla u\right)+(m-\Phi[u]) u .
$$

Equation (1.4) is degenerate if $m>1$ and singular if $0<m<1$. Equation (1.5) is degenerate if $p>2$ and singular if $1<p<2$. Only the cases $m>1$ and $p>2$ are considered with a few exceptions. All these equations are considered with the Dirichlet boundary condition which describes that the boundary is lethal to the species. Moreover, the methods in these papers are all based on the theory of Leray-Schauder degree. However, results on the quasilinear periodic parabolic equations with nonlocal terms and Neumann boundary conditions are few. In a recent paper [8], Wang and Yin considered the following periodic Neumann boundary value problem:

$$
\begin{aligned}
& \frac{\partial u}{\partial t}-\Delta u^{m}=(m-\Phi[u]) u, \quad(x, t) \in Q_{T}, \\
& \frac{\partial u}{\partial n}=0, \quad(x, t) \in \partial \Omega \times(0, T), \\
& u(x, 0)=u(x, T), \quad x \in \Omega,
\end{aligned}
$$

where $m>1$. By the parabolic regularized method and the theory of Leray-Schauder degree, they established the existence of nontrivial nonnegative periodic solutions.

Inspired by the work of [8], we consider the periodic solutions of the Neumann boundary value problem of a quasilinear parabolic equation with nonlocal terms. Compared with the Dirichlet boundary condition, the Neumann boundary condition causes an additional difficulty in establishing some a priori estimates. On the other hand, different from the cases of the Dirichlet boundary condition, an auxiliary problem for (1.1)-(1.3) is considered for using the theory of Leray-Schauder degree. We prove that this problem (1.1)-(1.3) admits a nontrivial nonnegative periodic solution, that is, the following theorem.

Theorem 1 If assumptions (A1), (A2), (A3) hold, then problem (1.1)-(3.3) admits a nontrivial nonnegative periodic solution $u \in L^{2}\left(0, T ; H^{1}(\Omega)\right) \cap C_{T}\left(\overline{Q_{T}}\right)$.

The article is organized in the following way. In Section 2, we give some necessary preliminaries including the auxiliary problem. In Section 3, we establish some necessary a priori estimations of the solutions of the auxiliary problem. Then we show the proof of the main result of this paper.

\section{Preliminaries}

In the paper, we assume that

(A1) $a_{i j}(\cdot, \cdot, u)=a_{j i}(\cdot, \cdot, u) \in C_{T}\left(\bar{Q}_{T}\right)$ and there exist two constants $0<\lambda \leq \gamma$ such that

$$
\lambda|\xi|^{2} \leq a_{i j}(x, t, u) \xi_{i} \xi_{j} \leq \gamma|\xi|^{2} \quad \text { for all }(x, t) \in Q_{T}, u \in R^{+} \text {and } \xi \in R^{n}
$$


where $C_{T}\left(\bar{Q}_{T}\right)$ is the class of functions which are continuous in $\bar{\Omega} \times \mathbb{R}$ and

$T$-periodic with respect to $t$. Furthermore, $a_{i j}(\cdot, \cdot, u)$ is continuous with respect to $u$.

(A2) $\Phi[\cdot]: L_{+}^{2}(\Omega) \rightarrow \mathbb{R}^{+}$is a bounded continuous functional satisfying

$$
\Phi[u] \leq C\|u\|_{L^{2}(\Omega)}^{2},
$$

where $C$ is a positive constant independent of $u, \mathbb{R}^{+}=[0,+\infty)$,

$L_{+}^{2}(\Omega)=\left\{u \in L^{2}(\Omega) \mid u \geq 0\right.$, a.e. in $\left.\Omega\right\}$.

(A3) $m(x, t) \in C_{T}\left(\bar{Q}_{T}\right)$ and satisfies that

$$
\underset{x \in \Omega}{\operatorname{essinf}} \frac{1}{T} \int_{0}^{T} m(x, t) d t>\gamma \lambda_{1}
$$

where $\lambda_{1}$ is the first eigenvalue of the Laplacian equation on $\omega$ with zero boundary and $\phi_{1}(x)$ is the corresponding eigenfunction.

Since the regularity follows from a quite standard approach, we focus on the discussion of weak solutions in the following sense.

Definition 1 A function $u$ is said to be a weak solution of problem (1.1)-(1.3), if $u \in$ $L^{2}\left(0, T ; H^{1}(T)\right) \cap C_{T}\left(\bar{Q}_{T}\right)$ and satisfies

$$
\iint_{Q_{T}}\left(-u \frac{\partial \varphi}{\partial t}+a_{i j}(x, t, u) D_{i} u D_{j} \varphi-(m-\Phi[u]) u \varphi\right) d x d t=0,
$$

for any $\varphi \in C^{1}\left(\bar{Q}_{T}\right)$ with $\varphi(x, 0)=\varphi(x, T)$.

In order to use the theory of Leray-Schauder degree, we introduce a map by considering the following auxiliary problem:

$$
\begin{aligned}
& \frac{\partial u_{\varepsilon}}{\partial t}-D_{i}\left((1-\tau) \gamma D_{i} u_{\varepsilon}+\tau a_{i j}(x, t, u) D_{j} u_{\varepsilon}\right)+\varepsilon u_{\varepsilon}=f, \quad(x, t) \in Q_{T}, \\
& \frac{\partial u_{\varepsilon}}{\partial n}=0, \quad(x, t) \in \partial \Omega \times(0, T), \\
& u_{\varepsilon}(x, 0)=u_{\varepsilon}(x, T), \quad x \in \Omega,
\end{aligned}
$$

where $\varepsilon$ is a sufficiently small positive constant, $\tau \in[0,1]$ is a parameter and $f \in C_{T}\left(\overline{Q_{T}}\right)$. Then we can define a map $u_{\varepsilon}=G(\tau, f)$ with $G:[0,1] \times C_{T}\left(\bar{Q}_{T}\right) \rightarrow C_{T}\left(\bar{Q}_{T}\right)$. Applying classical estimates (see [9]), we can see that $\left\|u_{\varepsilon}\right\|_{L^{\infty}\left(Q_{T}\right)}$ is bounded by $\|f\|_{L^{\infty}\left(Q_{T}\right)}$, and $u_{\varepsilon}$ is Hölder continuous in $Q_{T}$. Then, by the Arzela-Ascoli theorem, the map $G$ is compact. So, the map $G$ is a compact continuous map. Let $f\left(u_{\varepsilon}\right)=\left(m-\Phi\left[u_{\varepsilon}\right]\right) u_{\varepsilon}^{+}$, where $u_{\varepsilon}^{+}=\max \left\{u_{\varepsilon}, 0\right\}$, we can see that the nonnegative solution $u_{\varepsilon}$ of problem (2.2)-(2.4) is also a nonnegative fixed point of the map $u_{\varepsilon}=G\left(1,\left(m-\Phi\left[u_{\varepsilon}\right]\right) u_{\varepsilon}^{+}\right)$. So, we will study the existence of nonnegative fixed points of the map $u=G\left(1,\left(m-\Phi\left[u_{\varepsilon}\right]\right) u_{\varepsilon}^{+}\right)$instead of a nonnegative solution of problem (2.2)-(2.4). And the desired solution $u$ of (1.1)-(1.3) would be obtained as a limit point of $u_{\varepsilon}$.

\section{The proof of the main result}

First, by the same method as in [4], we can obtain the nonnegativity of the solutions of problem (2.2)-(2.4). 
Lemma 1 If a nontrivial function $u_{\varepsilon} \in C_{T}\left(\bar{Q}_{T}\right)$ solves $u_{\varepsilon}=G\left(1,\left(m-\Phi\left[u_{\varepsilon}\right]\right) u_{\varepsilon}^{+}\right)$, then

$$
u_{\varepsilon}(x, t)>0 \quad \text { for all }(x, t) \in \bar{Q}_{T} .
$$

In the following, we will show some $a$ priori estimates for the upper bound of a nonnegative periodic solution of problem (2.2)-(2.4). Here and below, we denote by $\|\cdot\|_{p}$ $(1 \leq p \leq \infty)$ the $L^{p}(\Omega)$ norm.

Lemma 2 For $\lambda \in[0,1]$, let $u(x, t)$ be a nonnegative periodic solution which solves $u_{\varepsilon}=$ $G\left(1, \lambda\left(m-\Phi\left[u_{\varepsilon}\right]\right) u_{\varepsilon}^{+}\right)$, then there exists a constant $K$ independent of $\lambda, \varepsilon$ such that

$$
\|u(t)\|_{\infty}<K
$$

where $u(t)=u(\cdot, t)$.

Proof Multiplying (2.2) by $u^{m+1}(m \geq 0)$ and integrating over $\Omega$, we have

$$
\frac{1}{m+2} \frac{d}{d t}\|u(t)\|_{m+2}^{m+2}+\frac{4(m+1)}{(m+2)^{2}}\left\|\nabla\left(|u(t)|^{\frac{m}{2}} u(t)\right)\right\|_{2}^{2} \leq\|m(x, t)\|_{L^{\infty}(\Omega \times(0, T))}\|u(t)\|_{m+2}^{m+2},
$$

and hence

$$
\frac{d}{d t}\|u(t)\|_{m+2}^{m+2}+C_{1}\left\|\nabla\left(|u(t)|^{\frac{m}{2}} u(t)\right)\right\|_{2}^{2} \leq C_{2}(m+2)\|u(t)\|_{m+2}^{m+2}
$$

where $C_{i}(i=1,2)$ are positive constants independent of $u$ and $m$. Assume that $\|u(t)\|_{\infty} \neq 0$ and set

$$
u_{k}(t)=|u(t)|^{\frac{m_{k}}{2}} u(t), \quad m_{k}=2^{k}-2(k=1,2, \ldots),
$$

then $m_{k}=2 m_{k-1}+2$. For convenience, we denote by $C$ a positive constant independent of $k$ and $m$, which may take different values. From (3.2), we obtain

$$
\frac{d}{d t}\left\|u_{k}(t)\right\|_{2}^{2}+C\left\|\nabla u_{k}(t)\right\|_{2}^{2} \leq C(m+2)\left\|u_{k}(t)\right\|_{2}^{2} .
$$

By using the Gagliardo-Nirenberg inequality, we have

$$
\left\|u_{k}(t)\right\|_{2} \leq C\left\|\nabla u_{k}(t)\right\|_{2}^{\theta}\left\|u_{k}(t)\right\|_{1}^{1-\theta},
$$

with

$$
\theta=\frac{N}{N+2} \in(0,1) .
$$

By inequalities (3.3), (3.4) and the fact that $\left\|u_{k}(t)\right\|_{1}=\left\|u_{k-1}(t)\right\|_{2}^{2}$, we obtain the following differential inequality:

$$
\begin{aligned}
\frac{d}{d t}\left\|u_{k}(t)\right\|_{2}^{2} & \leq-C\left\|u_{k}(t)\right\|_{2}^{\frac{2}{\theta}}\left\|u_{k}(t)\right\|_{1}^{\frac{2(\theta-1)}{\theta}}+C\left(m_{k}+2\right)\left\|u_{k}(t)\right\|_{2}^{2} \\
& \leq-C\left\|u_{k}(t)\right\|_{2}^{\frac{2}{\theta}}\left\|u_{k-1}(t)\right\|_{2}^{\frac{4(\theta-1)}{\theta}}+C\left(m_{k}+2\right)\left\|u_{k}(t)\right\|_{2}^{2} .
\end{aligned}
$$


Let

$$
\lambda_{k}=\max \left\{1, \sup _{t}\left\|u_{k}(t)\right\|_{2}\right\}
$$

we have

$$
\begin{aligned}
\frac{d}{d t}\left\|u_{k}(t)\right\|_{2}^{2} \leq & \left\|u_{k}(t)\right\|_{2}^{\frac{2\left(m_{k}+1\right)}{m_{k}+2}}\left\{-C\left\|u_{k}(t)\right\|_{2}^{\frac{2}{\theta}-\frac{2\left(m_{k}+1\right)}{m_{k}+2}} \lambda_{k-1}^{\frac{4(\theta-1)}{\theta}}\right. \\
& \left.+C\left(m_{k}+2\right)\left\|u_{k}(t)\right\|_{2}^{\frac{2}{m_{k}+2}}\right\} .
\end{aligned}
$$

By Young's inequality,

$$
a b \leq \epsilon a^{p^{\prime}}+\epsilon^{-\frac{q^{\prime}}{p^{\prime}}} b^{q^{\prime}}
$$

where $p^{\prime}>1, q^{\prime}>1, a>0, b>0, \epsilon>0$ and $\frac{1}{p^{\prime}}+\frac{1}{q^{\prime}}=1$. Set

$$
\begin{aligned}
& a=\left\|u_{k}(t)\right\|_{2}^{\frac{2}{m_{k}+2}}, \quad b=m_{k}+2, \quad \epsilon=\frac{1}{2} \lambda_{k-1}^{\frac{4(\theta-1)}{\theta}}, \\
& p^{\prime}=l_{k}=\frac{m_{k}+2}{\theta}-m_{k}-1=\left(m_{k}+2\right) \frac{N+2}{N}-m_{k}-1,
\end{aligned}
$$

then we obtain

$$
\left(m_{k}+2\right)\left\|u_{k}(t)\right\|_{2}^{\frac{2}{m_{k}+2}} \leq \frac{1}{2}\left\|u_{k}(t)\right\|_{2}^{\frac{2}{\theta}-\frac{2\left(m_{k}+1\right)}{m_{k}+2}} \lambda_{k-1}^{\frac{4(\theta-1)}{\theta}}+C\left(m_{k}+2\right)^{\frac{l_{k}}{l_{k}-1}} \lambda_{k-1}^{4 \frac{1-\theta}{\theta}} \frac{1}{k_{k}^{-1}} .
$$

Here we have used the fact that $p^{\prime}=l_{k}>r>1$ for some $r$ independent of $k$. In fact, it is easy to verify that

$$
\lim _{k \rightarrow \infty} l_{k}=+\infty
$$

Denoting

$$
a_{k}=\frac{l_{k}}{l_{k}-1}, \quad b_{k}=\frac{1-\theta}{\theta} \frac{4}{l_{k}-1},
$$

and combining (3.5) with (3.6), we have

$$
\frac{d}{d t}\left\|u_{k}(t)\right\|_{2}^{2} \leq\left\|u_{k}(t)\right\|_{2}^{\frac{2\left(m_{k}+1\right)}{m_{k}+2}}\left\{-C\left\|u_{k}(t)\right\|_{2}^{\frac{2}{\theta}-\frac{2\left(m_{k}+1\right)}{m_{k}+2}} \lambda_{k-1}^{\frac{4(\theta-1)}{\theta}}+C\left(m_{k}+2\right)^{a_{k}} \lambda_{k-1}^{b_{k}}\right\} .
$$

Then

$$
\left(m_{k}+2\right) \frac{d}{d t}\left\|u_{k}(t)\right\|_{2}^{\frac{2}{m_{k}+2}} \leq-C\left\|u_{k}(t)\right\|_{2}^{\frac{2}{\theta}-\frac{2\left(m_{k}+1\right)}{m_{k}+2}} \lambda_{k-1}^{\frac{4(\theta-1)}{\theta}}+C\left(m_{k}+2\right)^{a_{k}} \lambda_{k-1}^{b_{k}} .
$$

From the periodicity of $u_{k}(t)$, we know that there exists $t_{0}$ at which $\left\|u_{k}(t)\right\|_{2}$ reaches its maximum and thus the left-hand side of (3.8) vanishes. Then we obtain

$$
\left\|u_{k}(t)\right\|_{2} \leq\left\{C\left[\left(m_{k}+2\right)^{a_{k}} \lambda_{k-1}^{b_{k}+\frac{4(1-\theta)}{\theta}}\right]\right\}^{\frac{1}{\alpha_{k}}}
$$


where

$$
\alpha_{k}=\frac{2}{\theta}-\frac{2\left(m_{k}+1\right)}{m_{k}+2}=\frac{2 l_{k}}{m_{k}+2} .
$$

Therefore, we conclude that

$$
\left\|u_{k}(t)\right\|_{2} \leq\left\{C\left(m_{k}+2\right)^{a_{k}} \lambda_{k-1}^{b_{k^{+}} \frac{4(1-\theta)}{\theta}}\right\}^{\frac{1}{\alpha_{k}}}=\left\{C\left(m_{k}+2\right)^{a_{k}}\right\}^{\frac{m_{k}+2}{2 l_{k}}} \lambda_{k-1}^{\frac{2(1-\theta)\left(m_{k}+2\right)}{\left(l_{k}-1\right) \theta}} .
$$

Since $\frac{m_{k}+2}{\left(l_{k}-1\right) \theta}=\frac{1}{1-\theta}$ and $\frac{m_{k}+2}{2 l_{k}}$ and $\alpha_{k}$ are bounded, we get

$$
\left\|u_{k}(t)\right\|_{2} \leq C A^{k} \lambda_{k-1}^{2}
$$

where $A>1$ is a positive constant independent of $k$. Then we have

$$
\ln \left\|u_{k}(t)\right\|_{2} \leq \ln \lambda_{k} \leq \ln C+k \ln A+2 \ln \lambda_{k-1},
$$

thus

$$
\begin{aligned}
\ln \left\|u_{k}(t)\right\|_{2} & \leq \ln C \sum_{i=0}^{k-2} 2^{i}+2^{k-1} \ln \lambda_{1}+\ln A\left(\sum_{j=0}^{k-2}(k-j) 2^{j}\right) \\
& \leq\left(2^{k-1}-1\right) \ln C+2^{k-1} \ln \lambda_{1}+f(k) \ln A,
\end{aligned}
$$

or

$$
\left\|u_{k}(t)\right\|_{m_{k}+2} \leq\left\{C^{2^{k-1}-1} \lambda_{1}^{2^{k-1}} A f(k)\right\}^{\frac{2}{m_{k}+2}},
$$

where

$$
f(k)=k-2(k+1)-2^{k-1}+2^{k+1} .
$$

Letting $k \rightarrow \infty$, we obtain

$$
\|u(t)\|_{\infty} \leq C \lambda_{1} \leq C\left(\max \left\{1, \sup _{t}\|u(t)\|_{2}\right\}\right)
$$

Now, we just need to show the estimate of $\|u(t)\|_{2}$. Multiplying (2.2) by $u$ and integrating by parts over $Q_{T}$, by the periodicity of $u$, we have

$$
\iint_{Q_{T}} \lambda_{1}|\nabla u|^{2}+\varepsilon u^{2} d t d x \leq \iint_{Q_{T}} \lambda u^{2}(m-\phi[u]) d t d x,
$$

which implies that

$$
\iint_{Q_{T}} u^{2}(m-\phi[u]) d t d x \geq 0 .
$$


Let $M=\max _{(x, t) \in \overline{\mathrm{Q}}_{T}} m(x, t)$, by assumption (A2), we have

$$
\begin{aligned}
0 & \leq \iint_{Q_{T}} u^{2}(m-\phi[u]) d t d x \leq M \iint_{Q_{T}} u^{2} d t d x-\iint_{Q_{T}} u^{2} \phi[u] d t d x \\
& \leq M \iint_{Q_{T}} u^{2} d t d x-C \int_{0}^{T}\|u\|_{2}^{2} d t,
\end{aligned}
$$

that is,

$$
\int_{0}^{T}\|u\|_{2}^{4} d t \leq C \int_{0}^{T}\|u\|_{2}^{2} d t
$$

where $C$ is a positive independent of $\lambda$. By Young's inequality, we have

$$
\int_{0}^{T}\|u\|_{2}^{2} d t \leq \int_{0}^{T} \frac{1}{4 \varepsilon^{2}}+\varepsilon^{2}\|u\|_{2}^{4} d t .
$$

Combining with the above inequality, we have

$$
\left\|u_{k}(t)\right\|_{2} \leq C
$$

which together with (3.9) implies (3.1), and thus the proof is complete.

Corollary 1 There exists a positive constant $R$ independent of $\varepsilon$ such that

$$
\operatorname{deg}\left(I-G\left(1,\left(m-\Phi\left[u_{\varepsilon}\right]\right) u_{\varepsilon}^{+}\right), B_{R}, 0\right)=1
$$

where $B_{R}$ is a ball centered at the origin with radius $R$ in $L^{\infty}\left(Q_{T}\right)$.

Proof It follows from Lemma 2 that there exists a positive constant $R$ independent of $\lambda, \varepsilon$ such that

$$
u \neq G\left(1,\left(m-\Phi\left[u_{\varepsilon}\right]\right) u_{\varepsilon}^{+}\right), \quad \forall u \in \partial B_{R}, \lambda \in[0,1]
$$

So, the degree is well defined on $B_{R}$. From the homotopy invariance of the Leray-Schauder degree and the existence and uniqueness of the solution of $G(1,0)$, we can see that

$$
\begin{aligned}
\operatorname{deg}\left(1-G\left(1,\left(m-\Phi\left[u_{\varepsilon}\right]\right) u_{\varepsilon}^{+}\right), B_{R}, 0\right) & =\operatorname{deg}\left(1-G\left(1, \lambda\left(m-\Phi\left[u_{\varepsilon}\right]\right) u_{\varepsilon}^{+}\right), B_{R}, 0\right) \\
& =\operatorname{deg}\left(1-G(1,0), B_{R}, 0\right) \\
& =1 .
\end{aligned}
$$

The proof is completed.

Lemma 3 There exist constants $r_{0}>0$ and $\varepsilon>0$ such that for any $r<r_{0}, \varepsilon<\varepsilon_{0}, u=$ $G\left(\tau,(m-\Phi[u]) u^{+}+(1-\tau)\right)$ admits no nontrivial solution $u$ satisfying

$$
0<\|u\|_{L^{\infty}\left(Q_{T}\right)} \leq r
$$

where $r$ is a positive constant independent of $\varepsilon$. 
Homed et al. Boundary Value Problems 2013, 2013:34

Page 8 of 11

http://www.boundaryvalueproblems.com/content/2013/1/34

Proof By contradiction, let $u$ be a nontrivial fixed point of $u=G\left(\tau,(m-\Phi[u]) u^{+}+1-\tau\right)$ satisfying $0<\|u\|_{L^{\infty}\left(Q_{T}\right)} \leq r$. For any given $\phi(x) \in C_{0}^{\infty}\left(B_{\delta}\left(x_{0}\right)\right)$, multiplying (2.2) by $\frac{\phi^{2}}{u}$ and integrating over $Q_{T}^{*}=B_{\delta}\left(x_{0}\right) \times(0, T)$, we have

$$
\begin{gathered}
\iint_{Q_{T}} \frac{\partial u}{\partial t}\left(\frac{\phi^{2}}{u}\right) d t d x+\iint_{Q_{T}}(1-\tau) \gamma D_{i} u D_{i}\left(\frac{\phi^{2}}{u}\right) d t d x \\
\quad+\iint_{Q_{T}} \tau a_{i j}(x, t, u) D_{j} u D_{i}\left(\frac{\phi^{2}}{u}\right) d t d x \\
=\iint_{Q_{T}}\left(\phi^{2}(m-\varepsilon-\Phi[u])+(1-\tau) \frac{\phi^{2}}{u}\right) d t d x .
\end{gathered}
$$

By the periodicity of $u$, the first term on the left-hand side is zero. The second term on the left-hand side can be rewritten as

$$
\begin{aligned}
& \iint_{Q_{T}}(1-\tau) \gamma D_{i} u D_{i}\left(\frac{\phi^{2}}{u}\right) d t d x \\
& \quad=\iint_{Q_{T}}(1-\tau) \gamma D_{i} u D_{i}\left(\phi \cdot \frac{\phi}{u}\right) d t d x \\
& \quad=\iint_{Q_{T}}(1-\tau) \gamma|D \phi|^{2} d t d x-\iint_{Q_{T}}(1-\tau) \gamma u^{2}\left|D\left(\frac{\phi}{u}\right)\right|^{2} d t d x
\end{aligned}
$$

The third term of the left-hand side of equation (3.11) can be rewritten as

$$
\begin{aligned}
& \iint_{Q_{T}} \tau a_{i j}(x, t, u) D_{j} u D_{i}\left(\frac{\phi^{2}}{u}\right) d t d x \\
& \quad=\iint_{Q_{T}} \tau a_{i j}(x, t, u) D_{j} u D_{i}\left(\phi \cdot \frac{\phi}{u}\right) d t d x \\
& \quad=\iint_{Q_{T}} \tau a_{i j}(x, t, u) 2 D_{j} u D_{i}\left(\frac{\phi}{u}\right) d t d x-\iint_{Q_{T}} \tau a_{i j}(x, t, u)\left(\frac{\phi}{u}\right)^{2} D_{i}(u) D_{j}(u) d t d x .
\end{aligned}
$$

Then from (3.10), we obtain

$$
\begin{aligned}
& \iint_{Q_{T}}(1-\tau) \gamma|D \phi|^{2} d t d x-\iint_{Q_{T}}(1-\tau) \gamma u^{2}\left|D\left(\frac{\phi}{u}\right)\right|^{2} d t d x \\
& \quad+\iint_{Q_{T}} \tau a_{i j}(x, t, u) 2 D_{j} u D_{i}\left(\frac{\phi}{u}\right) d t d x-\iint_{Q_{T}} \tau a_{i j}(x, t, u)\left(\frac{\phi}{u}\right)^{2} D_{i}(u) D_{j}(u) d t d x \\
& \quad=\iint_{Q_{T}} \phi^{2}(m-\varepsilon-\Phi[u]) d t d x+\iint_{Q_{T}}(1-\tau) \frac{\phi^{2}}{u} d t d x .
\end{aligned}
$$

From assumption (A1), we can see that

$$
\begin{gathered}
\iint_{Q_{T}}(1-\tau) \gamma|D \phi|^{2} d t d x+\iint_{Q_{T}} \tau a_{i j}(x, t, u) 2 D_{j} u D_{i}\left(\frac{\phi}{u}\right) d t d x \\
-\iint_{Q_{T}} \phi^{2}(m-\varepsilon-\Phi[u]) d t d x
\end{gathered}
$$




$$
\begin{aligned}
= & \iint_{Q_{T}}(1-\tau) \frac{\phi^{2}}{u} d t d x+\iint_{Q_{T}}(1-\tau) u^{2}\left|D\left(\frac{\phi}{u}\right)\right|^{2} d t d x \\
& +\iint_{Q_{T}} \tau a_{i j}(x, t, u)\left(\frac{\phi}{u}\right)^{2} D_{i}(u) D_{j}(u) d t d x \\
\geq & \iint_{Q_{T}}(1-\tau) \frac{\phi^{2}}{u} d t d x+\iint_{Q_{T}}(1-\tau) \gamma u^{2}\left|D\left(\frac{\phi}{u}\right)\right|^{2} d t d x \\
& +\iint_{Q_{T}} \tau \lambda\left(\frac{\phi}{u}\right)^{2}|D(u)|^{2} d t d x \\
\geq & 0 .
\end{aligned}
$$

Since $\tau \in[0,1]$, we have

$$
\iint_{Q_{T}} \gamma|D \phi|^{2} d t d x-\iint_{Q_{T}} \phi^{2}(m-\varepsilon-\Phi[u]) d t d x \geq 0 .
$$

By an approaching process, we choose $\phi=\phi_{1}$, where $\phi_{1}$ is the eigenvector of the first eigenvalue $\lambda_{1}$ in (A3), and then we obtain

$$
\begin{aligned}
0 & \leq \iint_{Q_{T}} \gamma\left|D \phi_{1}\right|^{2} d t d x-\iint_{Q_{T}} \phi_{1}^{2}(m-\varepsilon-\Phi[u]) d t d x \\
& =-\iint_{Q_{T}} \gamma \phi_{1} \Delta \phi_{1} d t d x-\iint_{Q_{T}} \phi_{1}^{2}(m-\varepsilon-\Phi[u]) d t d x \\
& =\iint_{Q_{T}} \gamma \lambda_{1} \phi_{1}^{2} d t d x-\iint_{Q_{T}} \phi_{1}^{2}(m-\varepsilon-\Phi[u]) d t d x \\
& =\int_{\Omega} \phi_{1}^{2} \int_{0}^{T}\left(\gamma \lambda_{1}-m+\varepsilon+\Phi[u]\right) d t d x .
\end{aligned}
$$

Thus, there exists $x_{0} \in \Omega$ such that $\int_{0}^{T}\left(\lambda_{1}-m\left(x_{0}, t\right)+\Phi\left[u\left(x_{0}, t\right)\right]\right) d t \geq 0$, then

$$
\frac{1}{T} \int_{0}^{T} m\left(x_{0}, t\right) d t \leq \gamma \lambda_{1}+\varepsilon+\frac{1}{T} \int_{0}^{T} \Phi\left[u\left(x_{0}, t\right)\right] d t .
$$

From assumption (A2), we can see that

$$
\frac{1}{T} \int_{0}^{T} m\left(x_{0}, t\right) d t \leq \gamma \mu_{1}+\varepsilon+C r^{2}|\Omega|
$$

holds for any sufficiently small $r$ and $\varepsilon$, which is a contradiction to assumption (A3). The proof is complete.

Corollary 2 There exists a small positive constant $r<R$ which is independent of $\varepsilon, \tau$ such that

$$
\operatorname{deg}\left(I-G\left(1,\left(m-\Phi\left[u_{\varepsilon}\right]\right) u_{\varepsilon}^{+}\right), B_{r}, 0\right)=0
$$

where $B_{r}$ is a ball centered at the origin with radius $r$ in $L^{\infty}\left(Q_{T}\right)$. 
Proof Similar to Lemma 3, we can see that there exists a positive constant $0<r<R$ independent of $\varepsilon$ such that

$$
u_{\varepsilon} \neq F\left(\tau,\left(m-\Phi\left[u_{\varepsilon}\right]\right) u_{\varepsilon}^{+}+1-\tau\right), \quad \forall u \in \partial B_{r}, \lambda \in[0,1]
$$

So, the degree is well defined on $B_{r}$. By Lemma 3.3, we can easily see that $u=G(0,(m-$ $\left.\Phi[u]) u^{+}+1\right)$ admits no solution in $B_{r}$. Then, by the homotopy invariance of the LeraySchauder degree, we have

$$
\begin{aligned}
\operatorname{deg}\left(I-G\left(1,\left(m-\Phi\left[u_{\varepsilon}\right]\right) u_{\varepsilon}^{+}\right), B_{r}, 0\right) & =\operatorname{deg}\left(1-G\left(0,\left(m-\Phi\left[u_{\varepsilon}\right]\right) u_{\varepsilon}^{+}+1\right), B_{r}, 0\right) \\
& =0 .
\end{aligned}
$$

The proof is completed.

Now, we show the proof of the main result of this paper.

Proof of Theorem 1 Using Corollaries 1 and 2, we have

$$
\operatorname{deg}\left(1-G(f(\cdot)), B_{R} \backslash B_{r}, 0\right)=1,
$$

where $R$ and $r$ are positive constants and $R>r$. Problem (2.2)-(2.4) admits a nonnegative nontrivial solution $u_{\varepsilon}$ with $r \leq\left\|u_{\varepsilon}\right\|_{\infty} \leq R$. Combining with the regularity results [9] and a similar argument as in [10], we can prove that the limit function of $u_{\varepsilon}$ is a nonnegative nontrivial periodic solution of problem (1.1)-(1.3).

\section{Competing interests}

The authors declare that they have no competing interests.

\section{Authors' contributions}

$\mathrm{RH}$, JS and BW carried out the proof of the main part of this article, BW corrected the manuscript and participated in its design and coordination. All authors have read and approved the final manuscript.

\section{Author details}

${ }^{1}$ Department of Mathematics, Harbin Institute of Technology, Harbin, 150001, P.R. China. ${ }^{2}$ Department of Mathematics, College of Education, University of Tikrit, Tikrit, Iraq.

\section{Acknowledgements}

This work is partially supported by the National Science Foundation of China (11271100, 11126222), the Fundamental Research Funds for the Central Universities (Grant No. HIT. NSRIF. 2011006), the Natural Sciences Foundation of Heilongjiang Province (QC2011C020) and also by the 985 project of Harbin Institute of Technology.

Received: 26 November 2012 Accepted: 2 February 2013 Published: 21 February 2013

\section{References}

1. Allegretto, W, Nistri, P: Existence and optimal control for periodic parabolic equations with nonlocal term. IMA J. Math. Control Inf. 16, 43-58 (1999)

2. Calsina, A, Perello, C: Equations for biological evolution. Proc. R. Soc. Edinb. 125A, 939-958 (1995)

3. Rouchon, P: Universal bounds for global solutions of a diffusion equation with a nonlocal reaction term. J. Differ. Equ. 193, 75-94 (2003)

4. Zhou, Q, Huang, R, Ke, YY, Wang, YF: Existence of the nontrivial nonnegative periodic solutions for the quasilinear parabolic equation with nonlocal term. Comput. Math. Appl. 50, 1293-1302 (2005)

5. Huang, R, Wang, Y, Ke, Y: Existence of the non-trivial nonnegative periodic solutions for a class of degenerate parabolic equations with nonlocal terms. Discrete Contin. Dyn. Syst. 5, 1005-1014 (2005)

6. Ke, Y, Huang, R, Sun, J: Periodic solutions for a degenerate parabolic equation. Appl. Math. Lett. 22, $910-915$ (2009)

7. Zhou, Q, Ke, YY, Wang, YF, Yin, JX: Periodic p-Laplacian with nonlocal terms. Nonlinear Anal. 66, $442-453$ (2007) 
8. Yifu, W, Yin, J: Periodic solutions for a class of degenerate parabolic equations with Neumann boundary conditions. Nonlinear Anal., Real World Appl. 12, 2069-2076 (2011)

9. Ladyzenskaja, O, Solonnikov, V, Uraltseva, N: Linear and Quasilinear Equations of Parabolic Type. Translations of Mathematical Monographs, vol. 23. Am. Math. Soc., Providence (1968)

10. Wu, ZQ, Zhao, JN, Yin, JX, Li, HL: Nonlinear Diffusion Equation. World Scientific, Singapore (2001)

doi:10.1186/1687-2770-2013-34

Cite this article as: Hameed et al.: Periodic solution of a quasilinear parabolic equation with nonlocal terms and Neumann boundary conditions. Boundary Value Problems 2013 2013:34.

Submit your manuscript to a SpringerOpen ${ }^{\circ}$ journal and benefit from:

- Convenient online submission

- Rigorous peer review

- Immediate publication on acceptance

Open access: articles freely available online

- High visibility within the field

- Retaining the copyright to your article

Submit your next manuscript at $>$ springeropen.com 